\title{
The effects of electromagnetic waves emitted by the cell phones on the testicular tissue
}

\author{
Muhammet Ihsan Karaman ${ }^{1}$, Ali Murat Gökçe ${ }^{1}$, Orhan Koca ${ }^{1}$, Bilal Karaman ${ }^{1}$, Metin Ishak Öztürk ${ }^{1}$, \\ Necati Yurdakul ${ }^{2}$, Feriha Ercan ${ }^{2}$ \\ ${ }^{1}$ Haydarpasa Numune Training and Research Hospital, Department of Urology, Istanbul, Turkey; \\ 2 Marmara University School of Medicine, Department of Histology and Embryology, Istanbul, Turkey.
}

\begin{abstract}
Summary Objectives: Various risks have emerged in parallel to the rapidly increasing use of cell phones. Herein we studied the effects of cell phone emitted electromagnetic waves (EMW) on rat testes. Material and Methods: Twenty one adult male Albino rats were grouped into 3 groups each consisting of 7 rats. The first group was exposed to EMW on talk mode for 8 hours per day for 20 days and then their testes were extracted. The testes of the second group were extracted after 20 days of whole day EMW exposure. The third group was the control group. For the statistical analysis MannWhitney $U$ analysis was performed.

Results: At light microscopic examination of the testicular tissue, the existence of a high number of immature cells in the lumen of the seminiferous tubule in addition to the normal seminiferous tubules, besides irregular tubules with a reduction in the spermatogenic cell lines and tubules without lumen were observed in groups 1 and 2 . Histopathological alterations were scored as $0=$ none, $1=$ low, 2 =medium, 3 = serious. The average scores of the three groups were found to be $4.25 \pm 1.5$ for the group $1,4.33 \pm 3.9$ for the group 2 and $0.37 \pm 1.1$ for the group 3 respectively. As a result of the statistical evaluation, group 1 and group 2 had significantly higher scores than the control group $(p=0.001)$.

Conclusion: Infertility is one of the current problems of today due to a rapid increase in its incidence and cost. The negative effects of the EMWs on the testis should be taken into account and the necessary measures should be taken for prevention.
\end{abstract}

KEY WORDS: Cell phone; Electromagnetic waves; Testes.

Submitted 18 July 2014; Accepted 18 August 2014

\section{INTRODUCTION}

The risks of the electromagnetic fields on human health are increasing due to more intense use of new technologies in the daily life.

Particularly, the increasing use of cell phones in recent years and concordant widespread of the base stations had impact in threatening human health.
Many laboratory studies and epidemiological surveys have reported the correlation between the electromagnetic field exposure and severe health-related problems. Immunity, nervous system, neuro-endocrine system, cardiovascular system and blood parameters are affected from the electromagnetic fields (1-3).

The male partner is pathologic in nearly $50 \%$ of the infertile couples (4). First and foremost reason for this is the reduction in the quality and quantity of the ejaculated sperm. There are many factors that affect the sperm quality and quantity such as drugs, radiation, viral causes, genetic diseases.

As the sufficient exposure time period has not passed for conduction of community based studies regarding the cell phones and base stations, there are certain epidemiological challenges in determining the exposure of human communities objectively. It is known that the use of cell phones is more common in young and middleaged people who carry them in their pockets, close to the scrotal area. According to the information obtained from recent studies, we developed a rat model to investigate the effects of the cell phone emitted electromagnetic waves (EMWs) on the testes which are the most important organs of the reproduction system.

\section{Materials AND MethodS}

After ethics committee approval, in order to study the effects of the EMWs emitted by the cell phones on the testes of the rats, 21 adult male Wistar albino rats were divided into 3 groups. Every group of 7 rats was kept in standardized cages with the dimension of $40 \times 60 \mathrm{~cm}$, indoor lighting illuminated for 12 hours and left in dark for 12 hours, kept at temperature of $22 \pm 2^{\circ} \mathrm{C}$ and humidity ratio of $50 \pm 10 \%$, and with free pellet feed and easy access to water.

Cell phones were suspended $1 \mathrm{~cm}$ above the cover of the cage of group 1 and 2. Philips Genie 900 ${ }^{\circledR}$ (Singapore) cell phones having the highest specific absorption rate (SAR) value in the market $(1.52 \mathrm{~W} / \mathrm{kg})$ were used. Every day the cell phones were plugged in the charger for 24 hours and they remained on talk mode for 8 hours and standby mode for 16 hours. 
Group 1 rats were exposed to the cell phone EMWs emitted from talk mode for 8 hours and standby mode for 16 hours every day for 20 days and then their testes were extracted. Group 2 rats were exposed to the EMW emitted by talk mode for 8 hours and standby mode for 16 hours every day for 20 days followed by exposition to standby mode in the laboratory environment for 20 days and then their testes were extracted. The rats in the third group (control group) were not exposed and were fed in the standard laboratory environment for 20 days and then their testes were extracted. For the extraction of the testes of the rats, intraperitoneal ketamine hydrochloride $45 \mathrm{mg} \backslash \mathrm{kg}$ was injected for anaesthesia. Then, their testes were extracted. The testicular material after extraction was divided in two parts for light and electron microscopic examination.

\section{Light microscopic preparation}

Testes samples taken for the light microscopic examinations were fixed in 10\% formaldehyde, then they were incubated through rising alcohol solution and rinsed with toluene. The cross-sections with a thickness of approximately $5 \mu \mathrm{m}$ were stained with hematoxylin and eosin (H\&E) and examined with Olympus BX51 photomicroscope for general morphological evaluation. Three different criteria were taken into consideration. Intercellular connections, normal, loose cellular organization and eosinophilic cytoplasm, cellular damages such as pyknotic nucleus; abnormal formation of germ cells and Sertoli cells and disruption in the intercellular connections; very low number of germ cells and Sertoli cells with disrupted Sertoli cells alone or disrupted intercellular connections

Five identical areas of each cross-section were evaluated at x 100 magnification for histopathological scoring. Histopathological alterations were scored as $0=$ none, 1 = low, 2 = medium, 3 = serious. The scoring was made individually for each criterion and the total scores of the three criteria were used for comparison purpose.

Transmission Electron Microscopic Preparation

For the examination with transmission electron microscopy tissues were fixed in $2.5 \%$ phosphate buffered glutaraldehide solution (0.1 M, pH 7.4) and were incubated in $1 \%$ phosphate buffered osmium tetroxide solution, then were polymerized in drying oven at $600 \mathrm{C}$ in Epon 812 after dehydration with alcohol solutions at serial increasing concentrations. The thin cross-sections with approximately $60 \mathrm{~nm}$ were examined with the Jeol 1200 TEM (Tokyo, Japan) scanning electron microscope after contrasted via the uranyl acetate and lead cirate.

\section{Statistical analysis}

For the statistical analysis SPSS statistical program was used, and Mann-Whitney $U$ analysis method was performed. $\mathrm{P}<0.05$ ( $\mathrm{p}$ value lower than 0.05 ) was considered as statistically significant.

\section{RESULTS}

The light microscopic examination of the testicular tissue, revealed seminiferous tubules with spermatogenic cell series having a normal morphology in the control
Table 1.

Comparison between the mean scores of three groups.

\begin{tabular}{l|c|c|c|}
\hline & Average \pm SD & Median & Range \\
\hline Group 1 & $4.25 \pm 1.5$ & 4.5 & 3 \\
\hline Group 2 & $4.33 \pm 3.9$ & 4.5 & 9 \\
\hline Group 3 & $0.37 \pm 1.1$ & 0 & 3 \\
\hline P* & \multicolumn{3}{|c}{$\mathrm{P}<0.05$} \\
\hline $\begin{array}{l}\text { *Group 1 and group 2 } p>0.05 ; \text { Group 1 and group 3 } p=0.001 ; \\
\text { Group 2 and group 3 } p=0.001 \text {. }\end{array}$ \\
\hline
\end{tabular}

group (Group 3) (Figure 1A). At electron microscopic examination, the seminiferous tubules with spermatogenic series had a normal morphology (Figure 1B).

At the light microscopic examination of the testes of the group of rats that were examined after additional 20 days following the initial exposure to EMWs for 20 days (Group 2), besides normal seminiferous tubules, tubules with a reduced spermatogenic cell lines and those lacking lumen were observed. Immature spermatogenic cells were present in the seminiferous tubule lumens (Figure 1C). Electron microscopic examination revealed enlarged spaces between detected Sertoli cells and cells of spermatogenic series but there were several spermatid at the spermiogenesis process (Figure 1D).

At the light microscopic examinations of the group of rats exposed to EMWs for 20 days (Group 1), there was a high number of immature cells in the lumen of the seminiferous tubule and in addition to the normal appearing tubules also irregular tubules with reduced spermatogenic cell lines and tubules without lumen were observed (Figure 1E). At electron microscopic examination, enlarged spaces between the Sertoli cells and the spermatogenic cells as well as increased spermatid were observed (Figure 1F).

The average scores of each of the three groups were found to be $4.25 \pm 1.5$ for the group $1,4.33 \pm 3.9$ for the group 2 and $0.37 \pm 1.1$ for the group 3 respectively (Table 1). As a result of the statistical assessment made between the groups no significant difference was observed between the groups 1 and 2 ( $p>0.05$ ), but average scores of both group 1 and 2 were significantly higher $(\mathrm{p}=0.001)$ than the control group.

\section{Discussion}

With the progress in technology, human life became easier but every new technological product brings certain disadvantages threatening human health. Many products as hair dryer, microwave oven, television and computers, that make life easier, have negative impact on human health due to EMWs they emit.

One of recent and widely used EMW emitting device is the cell phone. The radiofrequency waves emitted by the cell phones are thought to cause many hazardous effects at cellular and molecular levels. Recent studies revealed that the radiofrequency waves emitted, especially from the third generation cell phones, might have effects on living organisms, immunological system, nervous sys- 


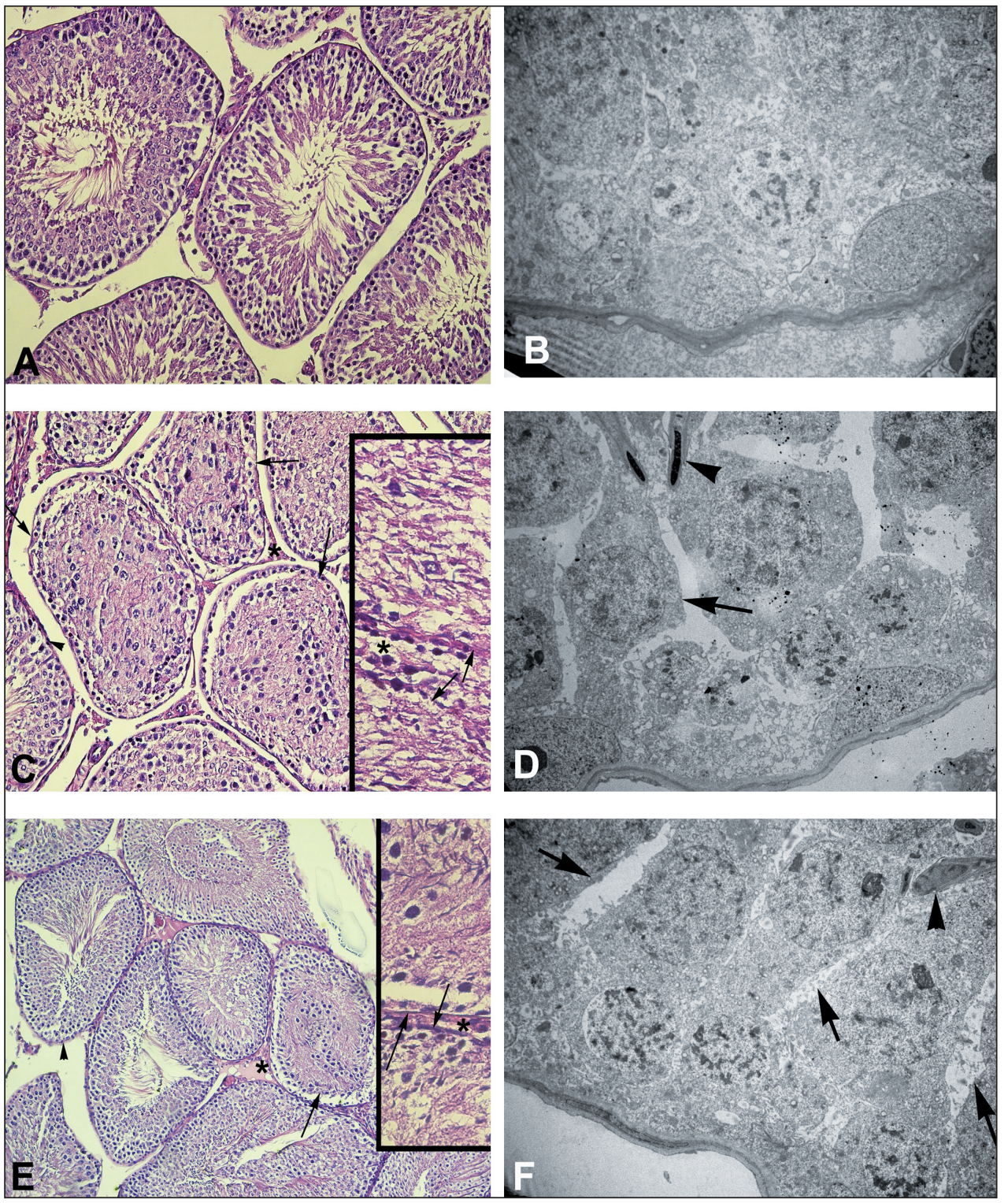

Figure 1.

Control group: Normal

morphology of seminiferous

tubules (A), Sertoli cells and

spermatogenic cells with

normal ultrastructure (B).

Group 2: Normal

morphology of seminiferous

tubules (arrow head),

irregular tubules with a

reduction in the

spermatogenic cell lines and

tubules without lumen

(arrow), interstitial zone $\left(^{*}\right)$

(C), spaces between the

Sertoli cells and

spermatogenic cells (arrow),

spermatid extending

towards the lumen (arrow

head) (D).

Group 1: Normal

morphology of seminiferous tubules (arrow head),

irregular tubules with a

reduction in the

spermatogenic cell lines and tubules without lumen (ok), interstitial zone $\left(^{\star}\right)(\boldsymbol{E})$, spaces between the Sertoli cells and spermatogenic cells (arrow), spermatid extending towards the lumen (arrow head) (F).

A, C, E; Haematoxylin-eosin stain, original magnification: $x$ 100, small pictures: x 200, B, D, F: Electron micrograph, original magnification: x 5000 .

tem, haematological function, cardiac functions, urinary system, growth and development as well as genetic structure (5). The EMWs emitted from the base stations and cell phones have damaging effects on tissues with two mechanisms. The thermal effects occur when electromagnetic energy absorbed by the body is converted into heat and the body temperature increases. This effect is balanced by the blood circulation. The non-thermal effects are manifested by changes in the brain functions, sleep and attention disorders, and headache (6). EMWs might also cause carcinogenesis (7)

Heat sensitivity of the testicular tissue was investigated in many studies (8). In the studies concerning the effects of EMWs on the testicular tissue, it was reported that high level of EMW exposition might increase the temperature of the testicular tissue causing serious pathologies such as degeneration, oedema, haemorrhage and reduction in spermatogenesis (8). The testicular damage we detected in this study might be due to such temperature increase. Dasdag et al. reported that the rectal temperature of the radiated mice were observed to be higher than the rectal temperature of the other mice (9). Akdag et al. also observed histopathological changes of mice testes following $9450 \mathrm{MHz}$ microwave radiation and thus concluded that long-term chronic microwave application might affect the epididymal sperm quantity, sperm morphology, weight of testes and epididymis, testicular and epididymal morphology of the mice and that the findings observed in the study increased depending on the duration of microwave application (10). Contrary to these studies, Dasdag et al. showed that the diameters of the EMW-treated rat seminiferous tubules were affected although sperm morphology and seminiferous tubules were not affected (9). The explanation of this result might be the insufficient period of daily exposure in such studies. Furthermore, as the cell phones used in our study had the highest SAR value (1.52) among all that available in the market, we conclude that microscopic findings of our cases might be due to this reason. Agarwal et al reported that the sperm mobility and sur- 
vivability of the samples exposed to EMW decreased noticeably, ROS levels increased and the score number of the ROS-TAC level decreased (11). The existence of tubules without lumen and an increase in the number of immature sperms were observed. Likewise, it was observed at electron microscopy that there are enlarged spaces between the Sertoli cells and the spermatogenic cells. Considering that the cell phones are positioned close to the testes during daily life, such findings might have an effect on infertility.

Statistically insignificant result of the light microscopic findings among the groups 1 and 2 point out that the effect of the EMWs on testis is irreversible. However, many spermatids in the spermiogenesis process were also detected in group 2 at the electron microscopic examination which gives the idea that such effects can be reversed in the longer time period. Considering the possible teratogenic and carcinogenic effects of EMWs due to their negative effects on chromosomes and DNA structure, unconscious use of cell phones by children and pregnant women might cause dangerous clinical outcomes in the long term. In order to take the necessary precautions, public information programs should be promoted. In our study some possible biases are improper size of the cell phones used with respect to the size of rats, and the lack of direct analysis of the effects on sperm motilities and fertility.

\section{Conclusion}

In conclusion, it should be kept in mind that cell phones might create a risk on human reproductive health taking into account of their widespread use and the location on the body where they are carried. EMWs emitted by cell phones cause histopathological changes of testicular tissue. Considering the long term effects of such changes, they could play an important role in the pathogenesis of important clinical diseases. Particularly special attention should be given to the damages that can be caused by the use of cell phones among risk groups (adults, children and pregnant women). Therefore, cell phones should be carried distant from the sensitive parts of the body such as testes. Unnecessary use of cell phones should be also prevented in order to minimize the deleterious effects.

\section{REFERENCES}

1 Eliyahu I, Luria R, Hareuveny R, et al. Effects of radiofrequency radiation emitted by cellular telephones on the cognitive functions of humans. Bioelectromagnetics. 2006; 27:119-26.

2. Hardell L, Mild KH, Carlberg M, Hallquist A. Cellular and cordless telephone use and the association with brain tumors in different age groups. Arch Environ Health. 2004; 59:132-7.

3. Koyu A, Cesur G, Ozguner F, et al. Effects of $900 \mathrm{MHz}$ electromagnetic field on TSH and thyroid hormones in rats. Toxicol Lett. 2005; 157:257-62

4. Dohle GR, Colpi GM, Hargreave TB, et al. EAU guidelines on male infertility. Eur Urol. 2005; 48:703-11.

5. Hardell L, Sage C. Biological effects from electromagnetic field exposure and public exposure standards. Biomed Pharmacother. 2008; 62:104-9.

6. Nakamura H, Matsuzaki I, Hatta K, et al. Nonthermal effects of mobile-phone frequency microwaves on uteroplacental functions in pregnant rats. Reprod Toxicol. 2003; 17:321-6.

7. Yakymenko I, Sidorik E. Risks of carcinogenesis from electromagnetic radiation of mobile telephony devices. Exp Oncol. 2010; 32:5460 .

8. Setchell BP. The Parkes Lecture. Heat and the testis. J Reprod Fertil. 1998; 114:179-94.

9. Dasdag S, Ketani MA, Akdag Z, et al. Whole-body microwave exposure emitted by cell phones and testicular function of rats. Urol Res. 1999; 27:219-23.

10. Akdag MZ, Çelik. MS, Ketani A, et al. Effect of Chronic LowIntesity Microwave Radiation on Sperm Count, Sperm Morphology, and Testicular and Epididymal Tissue of Rats. Electro-and Magnetobiology. 1999; 18:133-141.

11. Agarwal A, Desai NR, Makker K, et al. Effects of radiofrequency electromagnetic waves (RF-EMW) from cell phones on human ejaculated semen: an in vitro pilot study. Fertil Steril. 2009; 92:1318-25.

\section{Correspondence}

Muhammet Ihsan Karaman, MD

Ali Murat Gökçe, MD

Orhan Koca, MD (Corresponding Author)

drorhankoca@hotmail.com

Bilal Karaman, MD

Metin Ishak Öztürk, MD

Haydarpasa Numune Training and Research Hospital, Department of Urology,

Tibbiye cad. no:2 Üsküdar ZIP:34718 Istanbul, Turkey

Necati Yurdakul, MD

Feriha Ercam, MD

Marmara University School of Medicine,

Department of Histology and Embryology, Istanbul, Turkey 